NASA/CR-2006-214222

\title{
Two Point Space-Time Correlation of Density Fluctuations Measured in High Velocity Free Jets
}

Jayanta Panda

Ohio Aerospace Institute, Brook Park, Ohio 


\section{NASA STI Program . . . in Profile}

Since its founding, NASA has been dedicated to the advancement of aeronautics and space science. The NASA Scientific and Technical Information (STI) program plays a key part in helping NASA maintain this important role.

The NASA STI Program operates under the auspices of the Agency Chief Information Officer. It collects, organizes, provides for archiving, and disseminates NASA's STI. The NASA STI program provides access to the NASA Aeronautics and Space Database and its public interface, the NASA Technical Reports Server, thus providing one of the largest collections of aeronautical and space science STI in the world. Results are published in both non-NASA channels and by NASA in the NASA STI Report Series, which includes the following report types:

- TECHNICAL PUBLICATION. Reports of completed research or a major significant phase of research that present the results of NASA programs and include extensive data or theoretical analysis. Includes compilations of significant scientific and technical data and information deemed to be of continuing reference value. NASA counterpart of peer-reviewed formal professional papers but has less stringent limitations on manuscript length and extent of graphic presentations.

- TECHNICAL MEMORANDUM. Scientific and technical findings that are preliminary or of specialized interest, e.g., quick release reports, working papers, and bibliographies that contain minimal annotation. Does not contain extensive analysis.

- CONTRACTOR REPORT. Scientific and technical findings by NASA-sponsored contractors and grantees.
- CONFERENCE PUBLICATION. Collected papers from scientific and technical conferences, symposia, seminars, or other meetings sponsored or cosponsored by NASA.

- SPECIAL PUBLICATION. Scientific, technical, or historical information from NASA programs, projects, and missions, often concerned with subjects having substantial public interest.

- TECHNICAL TRANSLATION. Englishlanguage translations of foreign scientific and technical material pertinent to NASA's mission.

Specialized services also include creating custom thesauri, building customized databases, organizing and publishing research results.

For more information about the NASA STI program, see the following:

- Access the NASA STI program home page at http://www.sti.nasa.gov

- E-mail your question via the Internet to help@sti.nasa.gov

- Fax your question to the NASA STI Help Desk at 301-621-0134

- Telephone the NASA STI Help Desk at 301-621-0390

- Write to:

NASA STI Help Desk

NASA Center for AeroSpace Information 7121 Standard Drive Hanover, MD 21076-1320 
NASA/CR-2006-214222

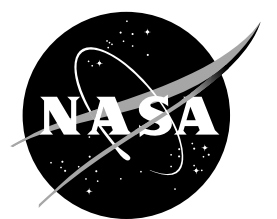

\section{Two Point Space-Time Correlation of Density Fluctuations Measured in High Velocity Free Jets}

Jayanta Panda

Ohio Aerospace Institute, Brook Park, Ohio

Prepared for the

44th Aerospace Science Meeting and Exhibit

sponsored by the American Institute of Aeronautics and Astronautics

Reno, Nevada, January 9-12, 2006

Prepared under Cooperative Agreement NCC3-1019

National Aeronautics and

Space Administration

Glenn Research Center

Cleveland, Ohio 44135 


\section{Acknowledgments}

This work was supported by NASA's Quiet Technology Demonstration program. The author is thankful to

Ray Lotenero of Gilcrest Company, for the help in building the optical setup; Kristie A. Elam of Jacobs Sverdrup, and Amy F. Mielke of NASA Glenn Research Center, for the help in the data acquisition system.

This work was sponsored by the Fundamental Aeronautics Program at the NASA Glenn Research Center.

Level of Review: This material has been technically reviewed by expert reviewer(s).

Available from

NASA Center for Aerospace Information 7121 Standard Drive

Hanover, MD 21076-1320
National Technical Information Service 5285 Port Royal Road Springfield, VA 22161 


\title{
Two Point Space-Time Correlation of Density Fluctuations Measured in High Velocity Free Jets
}

\author{
Jayanta Panda \\ Ohio Aerospace Institute \\ Brook Park, Ohio 44142
}

\begin{abstract}
Two-point space-time correlations of air density fluctuations in unheated, fully-expanded free jets at Mach numbers $\mathrm{M}_{\mathrm{j}}=0.95,1.4$, and 1.8 were measured using a Rayleigh scattering based diagnostic technique. The molecular scattered light from two small probe volumes of $1.03 \mathrm{~mm}$ length was measured for a completely non-intrusive means of determining the turbulent density fluctuations. The time series of density fluctuations were analyzed to estimate the integral length scale $\mathrm{L}$ in a moving frame of reference and the convective Mach number $\mathrm{M}_{\mathrm{c}}$ at different narrow Strouhal frequency (St) bands. It was observed that $\mathrm{M}_{\mathrm{c}}$ and the normalized moving frame length scale $\mathrm{L}^{*} \mathrm{St} / \mathrm{D}$, where $\mathrm{D}$ is the jet diameter, increased with Strouhal frequency before leveling off at the highest resolved frequency. Significant differences were observed between data obtained from the lip shear layer and the centerline of the jet. The wave numberfrequency transform of the correlation data demonstrated progressive increase in the radiative part of turbulence fluctuations with increasing jet Mach number.
\end{abstract}

\section{Introduction}

The statistical description of turbulence is usually based on the two point space time correlations of flow fluctuations (ref. 1). Various correlations commonly employed in turbulence modeling are subsets of information obtained from the general space-time correlation measurements. Therefore it is natural to see that the description of the noise sources in the acoustic analogy (ref. 2) or other schemes (ref. 3) depends on the space-time correlation of the turbulent field. All existing jet noise prediction schemes, that try to model the underlying physics, bank on experimental data to describe the spatial and the temporal variations of the correlation coefficients. The need for the correlation data was realized as soon as the Lighthill's celebrated work was published and hot-wire measurements by Laurence (ref. 4), Davies et al. (ref. 5) and others provided insight. Like the prior modeling efforts most of these experimental data treats turbulence as all random fluctuations with single length and time-scale describing fluctuations at all frequencies. It has been realized that further improvements in noise prediction can not be achieved without considering the differences at different frequency ranges. The large-scale/fine-scale approach of Tam et al. (ref. 6) and the frequency dependent scales approach of Harper-Bourne (ref. 7) are examples of this change in the modeling approach. The present experimental effort is motivated by this new approach. The goal is to determine frequency dependence of parameters such as convective velocity, integral length scales, and time scales that are measured via space-time correlation.

The first significant measurement of space-time correlation in free jets used hot wire probes (refs. 4 and 5); the jets under study were in the Mach number range of 0.2 to 0.55 . To avoid excessive interference from probe supports inserted into the flow, usually measurements were done only in the peripheral shear layer. Nevertheless, the observations made in these tests are still used in noise source modeling. Some of the significant observations are the following. (a) The integral length scale along the axial direction in a fixed frame of reference varies linearly with the downstream distance $\ell_{\mathrm{x}}=0.13 \mathrm{x}$ for $\mathrm{x} / \mathrm{D}<6$. (b) The radial length scale is nearly a third of the axial one: $\ell_{\mathrm{r}}=\ell_{\mathrm{x}} / 3$. (c) The radial variation of $\ell_{\mathrm{x}}$ is small. (d) The convective velocity $\mathrm{Uc}$ varies from $0.7 \mathrm{U}_{\mathrm{j}}$ at the inside edge of the shear layer to $0.3 \mathrm{U}_{\mathrm{j}}$ at the outside edge with around $0.65 \mathrm{U}_{\mathrm{j}}$ at the max shear location. $U_{\mathrm{j}}$ is the jet centerline velocity. (e) The 
integral time scale in a moving frame of reference, calculated from the time envelope of correlation with different probe separation, is inversely proportional to the local shear: $T_{\tau} \propto 1 /(\partial \mathrm{U} / \partial \mathrm{r})$, where $\mathrm{U}$ is the local axial velocity and $r$ is the radial direction. The integral length and time scales are defined later in the text. An interesting debate started by these early works in 1960s' was whether to use an exponential decay function or a Gaussian decay function to model the time variation of correlation. It has remained an issue even today (refs. 8 to 10). Since all of these observations were made in low speed jets and primarily in the lip shear layer, there is a need to determine variations in other regions of the jet and also in the practical Mach and Reynolds number range. For example, according to the observation (e), moving frame integral time scale becomes unphysical at the jet centerline where shear gradient is zero.

Early on Fisher and Davies (ref. 11) recognized the need for determining the frequency dependence of the turbulent scales. They band-pass filtered hot-wire signals before performing correlation. A drawback of this approach is that the filter's impulse response causes spurious oscillations in the filtered correlation data. The true temporal behavior of the correlations, especially at longer time delay, becomes difficult to isolate. The narrower the frequency band the more persistent is the oscillations. The present work takes a frequency domain approach used by Harper-Bourne (ref. 7) that is more conducive to investigating frequency dependent correlation.

The modern optical techniques such as LDV (refs. 12 and 13), PIV (ref. 9) and optical deflectometry (ref. 14) overcome many limitations of hot-wire probes. The advanced computations based on LES (ref. 15) and DNS (ref. 16) methodologies have also joined the force to determine turbulence scales, but their efforts are mostly limited to low Reynolds number flows and over limited frequency ranges. The present Rayleigh scattering based diagnostics allowed for a completely non-intrusive means of measuring turbulent fluctuations. There are no seed particles used in the technique, instead light scattered by the gas molecules are analyzed. In principle, the scattered light can be used to determine velocity, density and temperature fluctuations, however; only density fluctuations are reported in the present paper. Density and axial velocity fluctuations are found to be well correlated; hence the space-time correlations measured from the density fluctuations are expected to be similar to that of the axial component of velocity.

Additionally, noise sources can be described via any flow variable and for the supersonic jets density is easily measurable.

The present work is a part of a large database development project for validation of the CFD and CAA codes. The time averaged velocity, density and temperature profiles, density fluctuations spectra, far field noise spectra, and noise sources identified by direct correlations between far field noise and flow fluctuations were reported earlier in references 17, 18, and 19.

\section{Basics of Space-Time Correlation}

The two point space-time correlation is defined either in a fixed frame of reference or in a moving frame that is convected with the flow at velocity $U_{c}$. When a probe is kept at a fixed position of the jet the measured temporal fluctuations are in a fixed frame of reference. The direct correlation measurement from two such probes placed at $\mathbf{X}$ and $\mathbf{X}+\boldsymbol{\eta}$ ( $\boldsymbol{\eta}$ is the separation) provides fixed frame correlation. Note that in general, the probe separation can be in the axial, radial or azimuthal direction, however; for the following discussion separations only along the axial direction is assumed so that $\eta=\hat{i} \eta_{x}$ where $\hat{i}$ is the unit vector in the stream wise direction and $\eta_{x}$ is the stream wise component of $\eta$. For the present case of density fluctuation it is defined as the following:

$$
\left\langle\rho^{\prime *} \rho^{\prime}\right\rangle\left(\mathbf{X}, \eta_{\mathbf{X}}, \tau\right)=\frac{1}{\mathrm{~T}} \int_{0}^{\mathrm{T}} \rho^{\prime}(\mathbf{X}, \mathrm{t}) \rho^{\prime}\left(\mathbf{X}+\hat{\mathrm{i}} \eta_{\mathrm{X}}, \mathrm{t}+\tau\right) \mathrm{dt}
$$

Where, $\rho^{\prime}(\mathrm{t})$ is the fluctuating part of the instantaneous air density obtained by subtracting the average: $\rho^{\prime}(\mathrm{t})=\rho(\mathrm{t})-\bar{\rho}$, and $\tau$ is the correlation time. If the probes were allowed to move with the local convective 
velocity, the resulting correlations would have been in a moving frame. Since turbulent fluctuations are convected by the flow, the signal from a fixed probe is expected to show rapid fluctuations and is expected to de-correlate over smaller spatial and temporal separations: $\eta_{x}$ and $\tau$. However, if the effect of the convection is avoided by measurements in a moving frame, then correlation values represent the decay/growth and the distortion of eddies. This is expected to occur over longer separations. In spite of the modeling advantages (ref. 2), it is difficult to perform correlation measurements in a moving frame. Therefore, the moving frame properties are approximated from the fixed frame measurements. Note that in a moving frame the space-time correlations are related by convective velocity. Hence, if the experiment is conducted in a laboratory fixed frame the moving frame auto-correlation can be approximated by selectively choosing peaks in fixed-frame correlation (ref. 5):

$$
\left\langle\rho^{\prime *} \rho^{\prime}\right\rangle^{\mathrm{c}}(\mathbf{X}, \tau)=\left\langle\rho^{\prime *} \rho^{\prime}\right\rangle\left(\mathbf{X}, \eta_{\mathbf{x}}=\mathrm{U}_{\mathrm{c}} \tau, \tau\right)
$$

The superscript $\mathrm{c}$ denotes the moving frame correlation. The correlation values are normalized by the standard deviation of fluctuations $\rho_{\text {rms }}$ at the two measurement points and expressed as correlation coefficients:

$$
r\left(\mathbf{X}, \eta_{\mathbf{x}}, \tau\right)=\frac{\left\langle\rho^{\prime *} \rho^{\prime}\right\rangle\left(\mathbf{X}, \eta_{\mathbf{X}}, \tau\right)}{\rho_{\mathrm{rms}}^{\prime}(\mathbf{X}, 0) \rho_{\mathrm{rms}}^{\prime}\left(\mathbf{X}, \eta_{\mathrm{x}}\right)}
$$

The definition of the convective velocity has been a subject of some discussion in the past (Wills (ref. 20)). It is defined as the ratio of $\eta / \tau$ that maximizes the correlation coefficient $r$. The derivative can be taken with respect to either $\eta$ or $\tau$. The latter is usually the case for a fixed separation $\eta_{f}$; we look for $\tau_{\mathrm{c}}$ such that:

$$
\frac{\partial \mathrm{r}\left(\eta_{\mathrm{f}}, \tau\right)}{\partial \tau}=0, \quad \mathrm{U}_{\mathrm{c}}=\frac{\eta_{\mathrm{f}}}{\tau_{\mathrm{c}}}
$$

It also can be defined in terms of a fixed time delay $\tau_{\mathrm{f}}$ that maximizes the correlation (Willmarth and Wooldridge (ref. 21)): $\partial \mathrm{r}\left(\eta, \tau_{\mathrm{f}}\right) / \partial \eta=0$ and $\mathrm{U}_{\mathrm{c}}=\eta_{\mathrm{d}} / \tau_{\mathrm{f}}$. Since in both situations, the convection velocity was found to be somewhat dependent upon the choice of the fixed parameter Wills (ref. 20) introduced a global average. The present work follows the commonly used definition of equation 4 to calculate the convective velocity.

For a generalized model of space-time correlation that can be incorporated in the turbulence (noise source) modeling, there is a need to determine length and time scale of turbulent eddy. This is done via integral length and time scales (Townsend (ref. 1)). In a fixed frame of reference, the integral length scale $\ell$ and time scale $t_{\tau}$ are defined as:

$$
\ell(\mathbf{X})=\int_{0}^{\infty} \mathrm{r}\left(\mathbf{X}, \eta_{\mathbf{x}}, 0\right) \mathrm{d} \eta_{\mathrm{x}}, \quad \mathrm{t}_{\tau}(\mathbf{X})=\int_{0}^{\infty} \mathrm{r}(\mathbf{X}, 0, \tau) \mathrm{d} \tau
$$

As discussed earlier integral scales defined in a moving frame (length $\mathrm{L}$, time $\mathrm{T}_{\tau}$ ) provide better insights into the distortion of turbulence. This can be calculated from the moving frame auto-correlation function as follows: 


$$
\mathrm{T}_{\tau}(\mathbf{X})=\int_{0}^{\infty} \mathrm{r}^{\mathrm{c}}(\mathbf{X}, \tau) \mathrm{d} \tau=\int_{0}^{\infty} \mathrm{r}\left(\mathbf{X}, \eta_{\mathbf{x}}=\mathrm{U}_{\mathrm{c}} \tau, \tau\right) \mathrm{d} \tau, \quad \mathrm{L}(\mathbf{X})=\mathrm{T}_{\tau} \mathrm{U}_{\mathrm{c}}
$$

For a frozen pattern of turbulence, $r^{c}=1$ and $L=\infty$. However in realistic flows $r^{c} \rightarrow 0$ as $\eta_{x} \rightarrow \infty$ and both $\mathrm{L}$ and $\mathrm{T}_{\tau}$ are finite. As discussed earlier the moving frame scales are longer than those in a fixed frame, $\mathrm{T}_{\tau} \gg \mathrm{t}_{\tau}$.

To introduce frequency dependent scales, the correlation function in the equation (1) is Fourier transformed in time to obtain cross spectrum $\mathrm{G}_{\mathrm{x} \eta}$ :

$$
\mathrm{G}_{\mathrm{x} \eta}\left(\mathbf{X}, \eta_{\mathrm{x}}, \omega\right)=\int_{-\infty}^{\infty}\left\langle\rho^{\prime *} \rho^{\prime}\right\rangle\left(\mathbf{X}, \eta_{\mathrm{x}}, \tau\right) \mathrm{e}^{\mathrm{i} \omega \tau} \mathrm{d} \tau=\mathrm{G}_{\mathrm{x}} \mathrm{G}_{\eta}^{*}=\left|\mathrm{G}_{\mathrm{x} \eta}\right| \mathrm{e}^{-\mathrm{i} \Phi}
$$

Here $G_{x}$ and $G_{\eta}$ represent Fourier transform of density fluctuations at $\mathbf{X}$ and $\mathbf{X}+i \eta_{x}$, and the superscript * represents complex conjugate. The cross-spectral phase $\Phi(\omega)$ reflects the time shift $\tau_{\mathrm{c}}$ in fluctuations between the two measurement points and can be expressed as: $\Phi=\omega \tau_{c}$. The primary cause of the time shift is the convection of turbulence fluctuations by the mean flow $\tau_{\mathrm{c}}=\eta_{\mathrm{x}} / \mathrm{U}_{\mathrm{c}}$. Any distortion of the turbulent eddies over the separation distance is also manifested in the phase. However, for small probe separation the latter contribution is expected to be small. Therefore, the frequency dependent convection velocity can be estimated as:

$$
\mathrm{U}_{\mathrm{c}}(\mathbf{X}, \omega)=\frac{\eta_{\mathrm{x}} \omega}{\Phi}
$$

To reduce the measurement uncertainty, and to somewhat cancel out the contribution from the distortion of eddies, the convective velocity is calculated via averaging over multiple separation points.

Returning back to equation (7), after stripping away the phase part (and thereby the effect of convection), the remaining magnitude part of the cross spectrum is indicative of the change in correlation seen by the probes if one of them were convected with velocity $U_{c}$. This approximates the decay in correlation in a moving frame of reference. Hence the magnitude of the cross-spectrum can be used to estimate the integral length scale in a moving frame of reference. Towards this goal, the cross-spectrum needs to be normalized using the individual auto-spectra, and a coherence function is introduced:

$$
\widetilde{R}\left(\mathbf{X}, \eta_{x}, \omega\right)=\frac{G_{x \eta}}{\left(\left|G_{x}\right|^{2}\left|G_{\eta}\right|^{2}\right)^{1 / 2}}=\frac{\left|G_{x \eta}\right| e^{-i \Phi}}{\left(\left|G_{x}\right|^{2}\left|G_{\eta}\right|^{2}\right)^{1 / 2}} \text { where, }\left|G_{x}\right|^{2}=G_{x} G_{x}^{*},\left|G_{\eta}\right|^{2}=G_{\eta} G_{\eta}^{*}
$$

Harper-Bourne (ref. 7) used a coherence function which is slightly different from the above expression. If $\left|G_{x}\right|^{2} \sim\left|G_{\eta}\right|^{2}$ or magnitudes of the turbulence fluctuations are similar then:

$$
\widetilde{\mathrm{R}}\left(\mathbf{X}, \eta_{\mathrm{x}}, \omega\right) \approx \frac{\left|\mathrm{G}_{\mathrm{x} \eta}\right|}{\left|\mathrm{G}_{\mathrm{x}}\right|^{2}} \mathrm{e}^{-\mathrm{i} \Phi}=\mathrm{Re}^{-\mathrm{i} \Phi}
$$

$\mathrm{R}\left(\mathbf{X}, \eta_{\mathrm{x}}, \omega\right)$ is a simplified coherence spectrum (described as coherence modulus by Harper-Bourne) and is indicative of frequency dependent correlation coefficient. In general $\mathrm{R}$ is unity for perfect correlation and zero for complete de-correlation. A downside of the simplification is that in a growing shear layer with continuous turbulence production $\mathrm{R}$ can take values greater than unity. This was frequently 
encountered in the present data. The frequency dependent scales in a moving frame can now be defined as:

$$
L(\mathbf{X}, \omega)=\int_{0}^{\infty} \mathrm{R}\left(\mathbf{X}, \eta_{\mathbf{x}}, \omega\right) \mathrm{d} \eta_{\mathrm{x}}, \quad \mathrm{T}_{\tau}(\mathbf{X}, \omega)=\frac{\mathrm{L}(\mathbf{X}, \omega)}{\mathrm{U}_{\mathrm{c}}}
$$

These integral scales and other parameters defined above are measured in the present work.

A great utility of the space-time correlation data is that it allows for a direct determination of the part of turbulent fluctuations capable of radiating to the far field. Sound generation process involves a coupling between the fluctuations measured in the source region and the ambient medium. Most of the fluctuations in the source create only evanescence waves that decay down within a few diameters from the jet. Only a part of the fluctuating energy that attains the speed of sound along an observer direction in the ambient medium is ultimately radiated to the far-field (ref. 22). A turbulent eddy convected at subsonic speed and without any distortion does not radiate sound. However, distortions associated with growth and decay enables a subsonic eddy to radiate (ref. 23). To determine this radiative component of fluctuations, one needs to perform a wave number $(\mathrm{k})$ - frequency $(\omega)$ transform of the correlation data. Towards this end at first correlation data $\left\langle\rho^{\prime} * \rho^{\prime}\right\rangle\left(X, \eta_{\mathrm{X}}, \tau\right)$ are measured from a large number of separations. Subsequently, space-time Fourier transforms shows the distribution of energy in the k- $\omega$ space:

$$
\mathrm{S}_{\mathrm{k} \omega}(\mathbf{X}, \mathrm{k}, \omega)=\int_{-\infty}^{\infty} \int_{-\infty}^{\infty}\left\langle\rho^{\prime} * \rho^{\prime}\right\rangle\left(\mathbf{X}, \eta_{\mathrm{x}}, \tau\right) \mathrm{e}^{\mathrm{i} \omega \tau} \mathrm{e}^{\mathrm{ik} \eta_{\mathrm{x}} \mathrm{d} \tau \mathrm{d} \eta_{\mathrm{x}}}
$$

In the far field, away from the jet, $\mathrm{k}-\omega$ spectrum is a straight line $\omega /|\mathrm{k}|=\mathrm{c}_{\infty}$, where $\mathrm{c}_{\infty}$ is the speed of sound in the ambient medium. In contrast, due to the dispersive nature of turbulence, where fluctuations at a given frequency may have a range of convective velocity and therefore many different wave numbers $\mathrm{k}, \mathrm{k}-\omega$ spectrum inside the plume is significantly broadened. The part of the spectrum with $\omega /|\mathrm{k}| \geq \mathrm{c}_{\infty}$ is capable of coupling (radiating) to the far field at different observer angles. Note that the wave-number is a vector quantity with components along the axial, radial and tangential directions. In the present work the $\mathrm{S}_{\mathrm{k} \omega}$ spectra in axial wave-numbers were calculated for different jet Mach numbers and at different locations inside the plumes.

\section{Experimental Setup}

The experiments were performed in an unheated small jet facility at the NASA Glenn Research Center. A convergent nozzle was used to produce subsonic plumes and convergent-divergent nozzles were used for the Mach 1.4 and 1.8 plumes. All three nozzles had a nominal equivalent exit diameter of $\mathrm{D}=2.54 \mathrm{~cm}$. A detailed description of air density measurements using Rayleigh scattering principles can be found in reference 18. Fundamentally, Rayleigh scattering is the result of elastic scattering (no change in frequency between the incident and the scattered light, except for the Doppler shift) of light caused by molecules, such as the Oxygen and Nitrogen molecules, present in the air. The intensity of the scattered light is directly proportional to the molecular number density, and therefore the bulk density $\rho$ at the probe location. For a fixed composition gas, such as air, and for a fixed optical setup the changes in the scattered light intensity is directly related to the changes in the air density at the probe location. This intensity measurement principle is used to determine turbulent density fluctuations in the present setup. Measurements using the Rayleigh scattering principle do not require any seed particles, and therefore, the nuisances and the uncertainties involved in particle based measurement techniques are avoided. On the downside, to avoid large scattering from the contaminated particles special precautions have to be taken to cleanse the jet of dust particles. Reference 18 provides a detail description of the steps taken towards 
this goal. For the sake of brevity only the new part, created for the two beam setup, is described in the following.

The present optical setup around the nozzle facility is different from the earlier version used to measure single-point statistics. Figure 1 shows schematics of the facility and figure 2 shows a photograph of the facility. The optical setup consists of two beams crossing the jet plume in vertical and horizontal planes. The top view in figure 1(a) highlights the optics for the horizontal beam while that of the vertical beam is better seen in the side view of figure 1(b). Laser light scattered from a small region of each beam was collected using two separate sets of lenses oriented $90^{\circ}$ to the laser paths, and measured by photomultiplier tubes. The scattering angle is of little importance for density measurement; however; the setup was built with an ambition of simultaneously measuring density and velocity fluctuations from two different points in the jet. Since velocity measurement involves determination of the Doppler shift of the scattered light, the beam path and the collection direction are designed such that the same axial component of velocity could be measured by either setup.

Two mounting frames holding beam-forming optics, beam dump and collection optics for each beam were mounted on separate 2-axes traversing units. The traversing units allowed independent positioning of the probe volumes in an axial(x)-radial(r) plane. The light source is a continuous-wave, frequencydoubled $\mathrm{Nd}: \mathrm{VO}_{4}$ solid state laser operating at $532 \mathrm{~nm}$ wavelength. The laser head was placed at the bottom of the structural frame attached to the horizontal traversing unit. The primary beam was split into two parts by a 30 to 70 beam splitter. The weaker 30 percent part of the beam was passed directly via a focusing lens and a half-wave plate to form the horizontal beam. The horizontal probe volume was set at the focal point where the beam waist is about $0.16 \mathrm{~mm}$. The beam is ultimately dissipated in a beam dump. The second, 70 percent part of the primary beam was coupled to a multimode, 100 micron core diameter optical fiber and passed to the top of the frame holding optics for the vertical probe volume. A combination of two lenses, 80 and $300 \mathrm{~mm}$ focal lengths, focused the light emerging from the fiber to a $0.38 \mathrm{~mm}$ waist, which constituted the vertical probe volume. The collection optics for each unit were made of a combination of 160 and $300 \mathrm{~mm}$ focal lengths and $80 \mathrm{~mm}$ diameter lenses, which focused the collected scattered light from each probe volumes on a $0.55 \mathrm{~mm}$ core diameter multimode fiber. The fiber diameter fixed the probe volume length (extent of the laser beams imaged on the fiber) to $1.03 \mathrm{~mm}$.

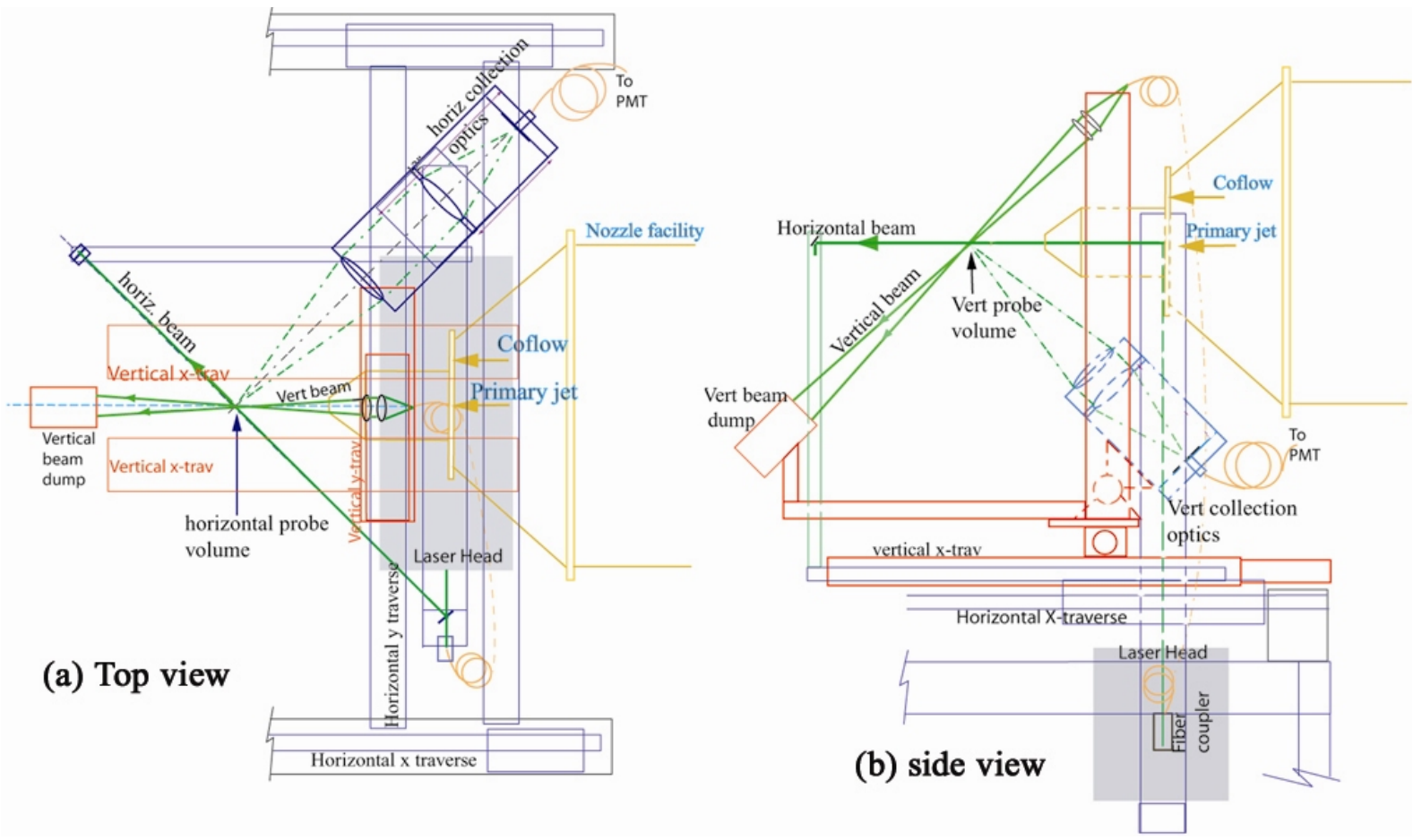

Figure 1.-Schematic of the facility. 


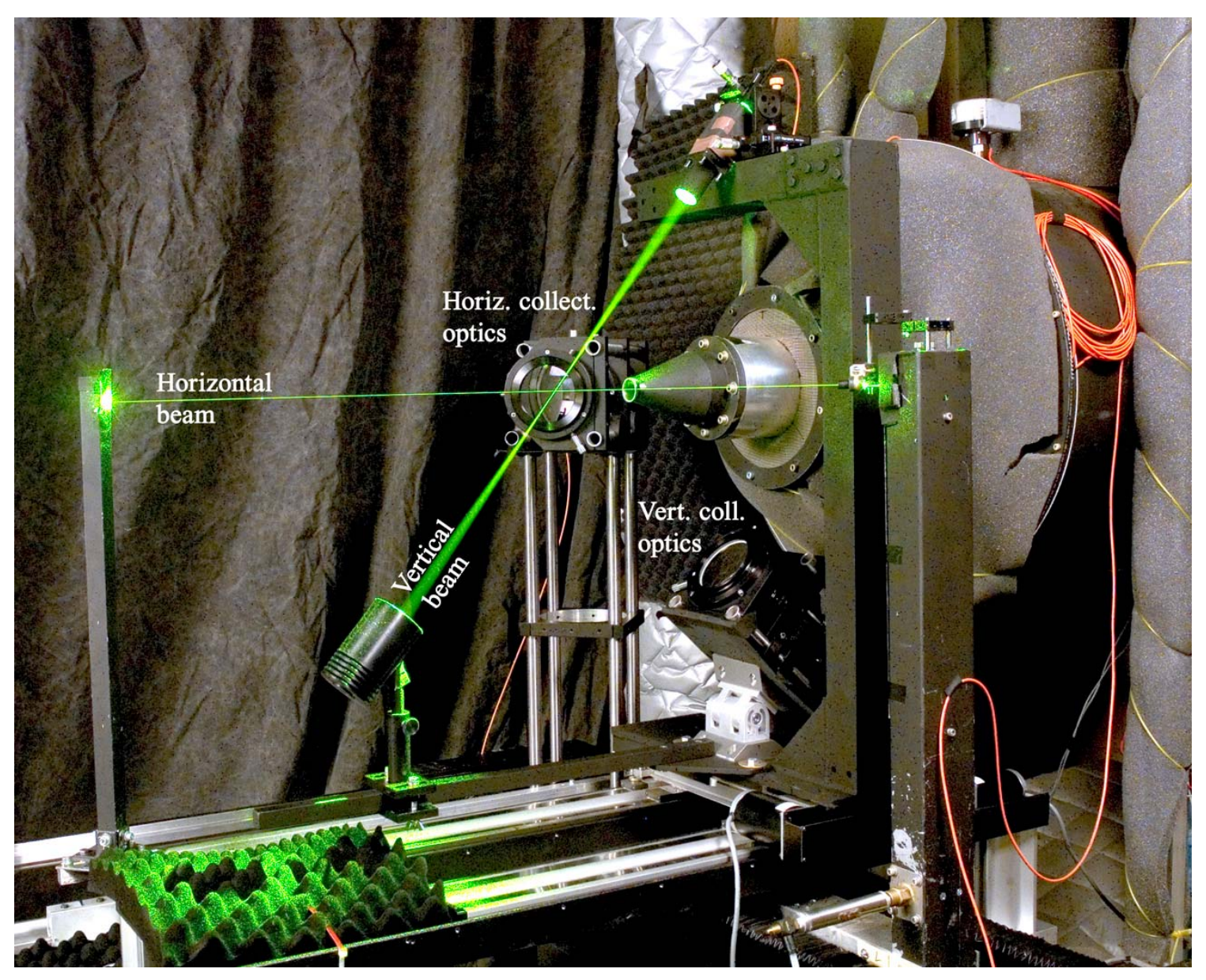

Figure 2.-Photograph of the facility.

Attention was paid to minimize vibration on the optical components by the high noise level produced by the jet. The laser head was enclosed in a semi-anechoic chamber and mounting frames were placed upstream while the laser beam propagated downstream.

Since the intensity of the Rayleigh scattered light is polarization dependent, the horizontal beam was vertically polarized to maximize scattering towards the horizontal collection optics. The vertical beam was passed through a multi-mode fiber which scrambled the incident polarization, and therefore; scattering from half of the vertical beam was effectively collected by the vertical collection optics. The 30 to 70 splitting of incident laser power was guided by the desire to achieve nearly the same scattering power at the two collection setups. Indeed at the end, when the probe volumes were separated, similar rates of photo-electron counts were achieved. However, when the beams were made to overlap the differences in polarizations caused the photon arrival rate to the horizontal collection optics to exceed that towards the vertical.

The scattered light, collected by the optical fibers, was passed to an adjoining room where two photomultiplier tubes and photo-electron counting electronics were kept (not shown in figs. 1 and 2). According to the Rayleigh scattering principle, for a fixed molecular composition gas (air in this instance), the intensity of the scattered light is directly proportional to the local air density. Therefore, the photoelectron arrival rate is related to the air density at the probe volume. The proportionality constant was determined through an in-situ calibration (ref. 18). It was performed in the plume of the convergent nozzle operated in the subsonic Mach numbers. At each operating condition the photon arrival rate was counted and the jet density was calculated using isentropic relations. Subsequently, a straight line was fit through the data to determine the proportionality constants $\mathrm{a}$ and $\mathrm{b}$. 


$$
\mathrm{N}=(\mathrm{a} \rho+\mathrm{b}) \Delta \mathrm{t}
$$

The additional constant $b$ is needed to account for the room light and stray scattered laser light. Since two counters were used, two sets of calibration constants $a_{1}, b_{1}$, and $a_{2}, b_{2}$ were calculated.

As described earlier, the cross correlation data are normalized by the auto-correlation at the reference location where both beams overlap. To avoid the electronic shot noise contribution (this will be described later in the text) auto-spectra are calculated by cross-correlating counts from two PMTs with overlapped probe volumes. Since the effective laser power nearly doubles when the beams overlap, a new set of calibration constants were needed to convert the photo-electron arrival rate to physical density numbers. Therefore, a total of four sets of calibration constant were calculated: two sets (one for each beam) with separated probe volumes and two with overlapped probe volumes. Overlapping the probe volumes involved maximizing the count rates by trial and error. This was achieved relatively easily at one point in the plume. However; due to a slight misalignment of the two traversing units, a problem appeared in maintaining the perfection at all other locations. The misalignment was small: few tenths of a millimeter over the traversing range, yet sufficient to cause a drift in calibration. Therefore, the overlapped probe calibration was adjusted based on the known density (measured by one of the separated probes) at the overlapped point.

The independent $\mathrm{x}-\mathrm{r}$ traversing units allowed arbitrary separation of the probe volumes. The minimum separation, that avoided "cross-talk" between the measurement points, was determined by the diameter $(0.55 \mathrm{~mm})$ of the optical fibers onto which the beams were imaged, the scattering angle $\left(90^{\circ}\right)$, and the probe volume diameters $(0.16$ and $0.38 \mathrm{~mm})$. The laser beam profile, at the focal point, is Gaussian and the diameters simply refer to the full width at half maxima of this profile. The tail of the laser beam extends beyond the prescribed diameter. Hence, it was found that the minimum separation for which independent measurements by the two probe volumes can be achieved is about $2 \mathrm{~mm}(0.08 \mathrm{D})$.

Returning back to the signal processing, to resolve time variation in density, photo-electron counting was performed in a series of contiguous time bins typically of $\Delta \mathrm{t}=8192 \mathrm{~ns}$ duration (the bin duration was changed for different Mach number jets). This produced an effective sampling rate of $122 \mathrm{~K} \mathrm{samples/sec.} \mathrm{The}$ photon counting process also introduces an effective low-pass filter whose description can be found in reference 18 . Usually two long records, each $n_{\text {tot }}=5,120,000$ points long, were collected for every measurement. The standard deviation of density fluctuations at each probe location was estimated from the power spectrum of the photon counts. The average values need to be subtracted from the individual strings, $\mathrm{N}_{\mathrm{i}}^{\prime}=\mathrm{N}_{\mathrm{i}}-\mathrm{N}_{\mathrm{av}}(\mathrm{i}=0,1,2, \ldots \mathrm{n}-1)$, before the power spectrum is calculated:

$$
\mathrm{P}_{\mathrm{N}^{/ 2}}\left(\mathrm{f}_{\mathrm{l}}\right)=2\left|\mathrm{~F}_{\mathrm{N}^{\prime}}(\mathrm{l})\right|^{2}, \quad \mathrm{f}_{1}=\frac{1}{\mathrm{n} \Delta \mathrm{t}} \quad \mathrm{l}=0,1,2, \ldots \ldots \frac{\mathrm{n}}{2}-1 \quad \text { where, } \mathrm{F}_{\mathrm{N}^{\prime}}(1)=\frac{1}{\mathrm{n}} \sum_{\mathrm{i}=0}^{\mathrm{n}-1} \mathrm{~N}_{\mathrm{i}}^{\prime} \exp \left(-\mathrm{j} \frac{2 \pi \mathrm{i} 1}{\mathrm{n}}\right)
$$

Here, $f_{1}$ is the frequency associated with each line. The Welch method of modified Periodograms (ref. 25) was used to calculate the spectral density. Each long record was divided into small segments of $n=512$ data points. The adjacent segments were overlapped by 50 percent. The modified periodograms of corresponding segments from the two PMTs were calculated and then used to determine local estimates of spectral density. All local estimates were averaged to obtain the final spectral density. The Power spectrum of the air density fluctuations requires multiplication by calibration constants:

$$
\mathrm{P}_{\rho^{/ 2}}\left(\mathrm{f}_{1}\right)=\frac{1}{\mathrm{a}^{2} \Delta \mathrm{t}^{2}} \mathrm{P}_{\mathrm{N}^{/ 2}}\left(\mathrm{f}_{1}\right)
$$

The problem with this straightforward method is that the calculated spectrum becomes overwhelmed from the electronic shot noise, an unavoidable consequence of low light level measurements. Shot noise poses the fundamental uncertainty in all light intensity measurements. Following Parseval's identity: 


$$
\sum_{1=0}^{\frac{n}{2}-1} P_{N^{/ 2}}\left(f_{1}\right)=\sigma_{N}^{2}=\sigma_{s h}^{2}+\sigma_{\rho}^{2} .
$$

In other words, the mean-square calculated from the spectrum $\sigma_{\mathrm{N}}^{2}$ is a sum of shot noise $\sigma_{\mathrm{sh}}^{2}$ and desired air density fluctuations $\sigma_{\rho}^{2}$. An estimate of the shot noise contribution can be obtained by noting that it is white noise and contributes equally to all frequency bins. The constant shot noise floor was estimated as the average of spectral density values at the highest end of the resolved frequency: of 55 to $61 \mathrm{KHz}$ (Nyquist limit):

$$
\mathrm{S}_{\text {shot }}=\frac{1}{6000} \int_{\mathrm{f}=55000}^{61000} \mathrm{P}_{\mathrm{N}^{/ 2}} \mathrm{df} \text {. }
$$

The energy from turbulent fluctuations is expected to be small over this frequency range. Since $\sigma_{\mathrm{sh}}^{2}=\int S_{\text {shot }} \mathrm{df}$, the standard deviation of density fluctuations is:

$$
\rho_{\mathrm{rms}}=\frac{1}{\mathrm{a}^{2} \Delta \mathrm{t}^{2}} \sqrt{\sum_{\mathrm{l}=0}^{\frac{\mathrm{n}}{2}-1}\left[\mathrm{P}_{\mathrm{N}^{/ 2}}-\mathrm{S}_{\mathrm{shot}}\right]}
$$

Individual power spectral density and shot-noise floors in PMT spectra are shown in figure 3(a). The data presented are in power spectral density (rather than power spectra) where the power in the individual bins are divided by the line width

$$
\mathrm{G}_{\mathrm{X}}^{2}\left(\mathbf{X}, \mathrm{f}_{1}\right)=\frac{\Delta \mathrm{t} \mathrm{P}_{\rho^{\prime 2}}}{2 \mathrm{n}},
$$

and frequency is normalized to Strouhal number $S t=f D / U_{j} ; U_{j}$ is the velocity in the potential core. The crossspectral calculation followed a similar path where the Fourier transform of one data series was multiplied by the complex conjugate (indicated by ${ }^{\mathrm{a}} *$ in the following equation) of the other:

$$
\mathrm{G}_{\mathrm{x} \eta}\left(\mathbf{X}, \eta_{\mathrm{x}}, \mathrm{f}_{1}\right)=\frac{\left(\mathrm{F}_{\mathrm{N}_{1}^{\prime}}(1) \mathrm{F}_{\mathrm{N}_{2}^{\prime}}^{*}(1)\right)}{\mathrm{a}_{1} \mathrm{a}_{2} \Delta \mathrm{tn}},
$$

The magnitude of the cross-spectral density is shown in figure 3(a) and phase in figure 3(c). The frequency $\mathrm{f}$ defined in this equation is related to the circular frequency in equation (7) via $\omega=2 \pi f$. The cross-spectral function, for the discretely sampled data, defined in the above equation is the stepping stone towards calculating correlation parameters defined in the Introduction. For example, the magnitudes of cross-spectra from a set of data with different separation $\eta_{\mathrm{x}}$ is used to calculate frequency dependent integral length scales while the phase variation is used for determining the convective velocity.

Notice that when the probe volumes are coinciding, i.e., $\eta=(0,0,0)$ the cross-spectrum becomes the auto-spectrum of the density fluctuations at the probe location $\mathbf{X}$. Interestingly this is the true auto-spectrum without the influence of electronic shot noise. Shot noise from two photo-multiplier tubes are uncorrelated; hence a cross-correlation between the two signals rejects this uncommon part, bringing out the desired 

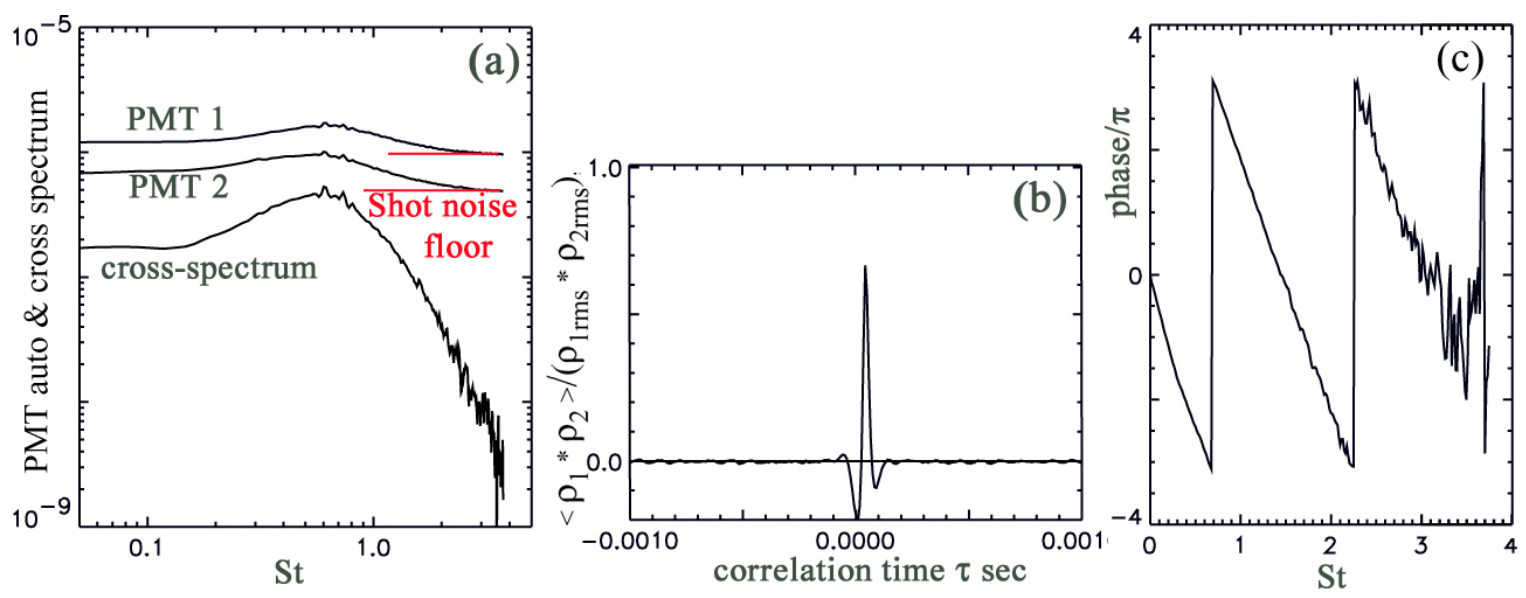

Figure 3.- (a) Auto-spectrum of individual PMT signal and magnitude of density cross-spectrum (b) cross-correlation and (c) cross-spectral phase in $\mathrm{Mj}=1.4$ jet with one probe at $(3.0 \mathrm{D}, 0.5 \mathrm{D})$ and the other at $(3.5 \mathrm{D}, 0.5 \mathrm{D})$.

spectrum of air density fluctuations. In reality, due to a finite averaging time, a noise floor remains as seen in the cross-spectrum presented in figure 3(a). This floor ultimately limits the usable frequency range for the correlation study. The cross-correlation was calculated via inverse Fourier transform $\mathrm{F}^{-1}$ of the crossspectrum:

$$
\left\langle\rho_{1} * \rho_{2}\right\rangle\left(\mathbf{X}, \eta_{\mathrm{x}}, \tau\right)=\frac{\mathrm{F}^{-1}\left(\mathrm{~F}_{\mathrm{N}_{1}^{\prime}}(1) \cdot \mathrm{F}_{\mathrm{N}_{2}^{\prime}}^{*}(1)\right)}{\mathrm{a}_{1} \mathrm{a}_{2} \Delta \mathrm{t}^{2}} \quad 1=0, \pm 1, \pm 2, \ldots \ldots \pm \frac{\mathrm{n}}{2}-1
$$

Figure 3(b) shows one such cross correlation plot. Following is a discussion of the uncertainty in the normalized correlation coefficients.

The cross-correlation process mostly eliminated contribution from the electronic shot noise. Additionally the large data sets used for calculation produced fairly converged statistics. However, the correlation values are normalized by individual standard deviations, $\frac{\left\langle\rho_{1} * \rho_{2}\right\rangle\left(\mathbf{X}, \eta_{\mathbf{x}}, \tau\right)}{\rho_{1 \mathrm{rms}}^{\prime}(\mathbf{X}) \rho_{2 \mathrm{rms}}^{\prime}\left(\mathbf{X}, \eta_{\mathbf{x}}\right)}$ which are the primary sources of uncertainty in the presented form. Calculation of the standard deviation of density fluctuations required shot noise subtraction as described earlier. The subtraction process produces estimation of the standard deviation within \pm 5 percent uncertainty. Hence, a maximum uncertainty level of \pm 10 percent is expected on the quoted normalized correlation values. This large uncertainty supersedes all other sources, such as the contamination from stray particles, numerical convergence error etc. It is also expected that the highest uncertainties are present in the lowest Mach number condition where the absolute value of density fluctuation is the lowest. The uncertainty level decreases with an increase in the jet Mach number.

\section{Results and Discussion}

Profiles of time averaged density and standard deviation of density fluctuations for the same jets under study were reported earlier in reference 18, and will not be repeated in this paper. The space-time correlation data were measured from many different locations in the plumes. Figure 4 shows distributions of the density fluctuations in the plumes and also identifies reference locations about which correlation data were obtained. The reference position signifies the location of the fixed probe volume. The second probe volume was mostly separated along the axial direction and, in a few cases, along the radial direction. Figure 4 demonstrates that 


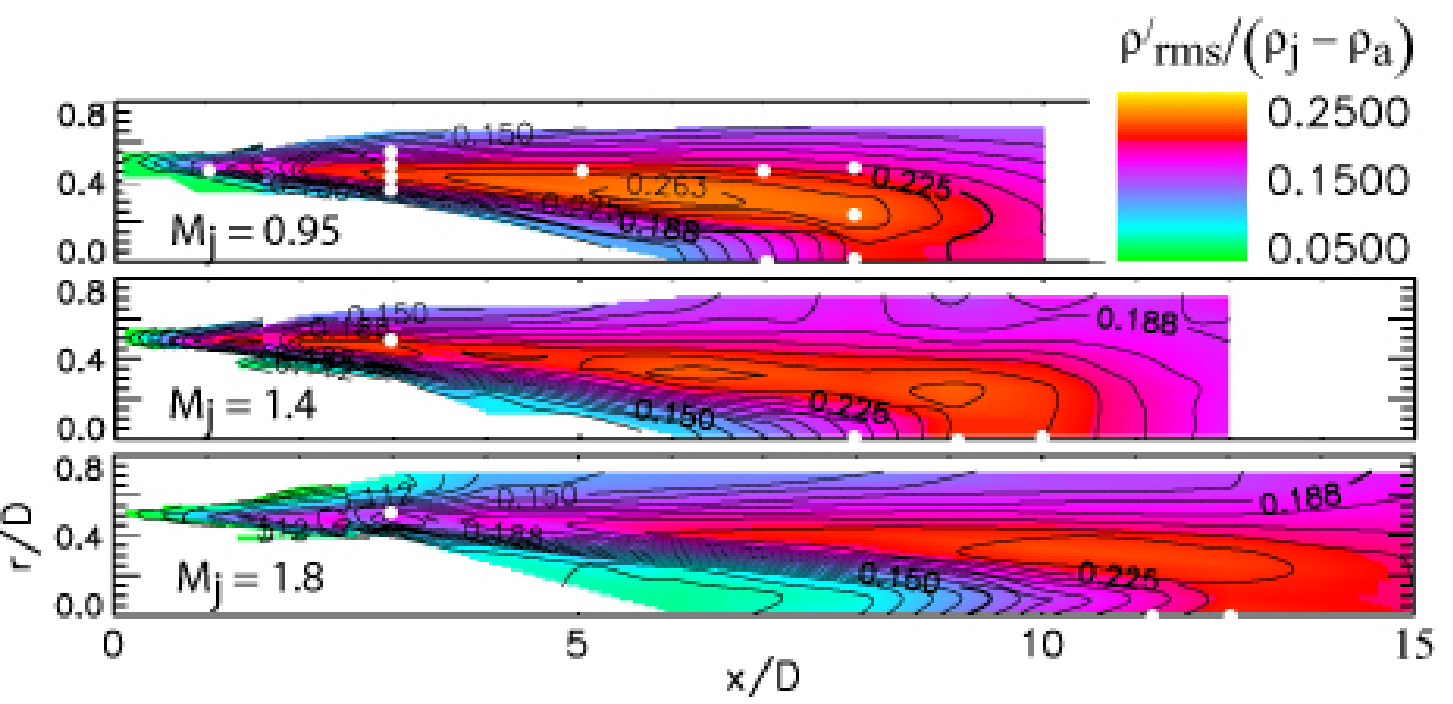

Figure 4.-Distribution of normalized density fluctuations in the indicated Mach number jets. The white dots indicate reference positions about which space-time correlations were measured.

an increase in the jet Mach number causes a progressive reduction in the growth rate of the lip shear layer which manifests in a lengthening of the potential core. Note that jets under study are unheated, i.e., the total temperature of the plume is the same as that of the ambient. The change in density is a manifestation of the compressibility effect. Therefore, the density fluctuations discussed in this paper are a reflection of the fluctuating compressibility which is expected to be closely related to noise sources.

The bulk of the figures presented in this paper are from two sets of reference points. For the first set, the reference point was kept fixed at the lip line and three diameters from the nozzle exit $\mathbf{X}(3.0 \mathrm{D}, 0.5 \mathrm{D})$. For the second set the reference point was placed at the centerline and three different axial positions $\mathrm{x} / \mathrm{D}=8,10$, and 12 for the three plumes of $\mathrm{M}_{\mathrm{j}}=0.95,1.4$, and 1.8, respectively. The difference in the axial location is a reflection of the increase in the potential core length $\mathrm{Lp}$, and nominally represents an equal distance of $\sim 1.2$ Lp.

Figures 5(a) and (c) shows two sets of normalized correlation plots for the reference probe located at the lip shear layer and at the centerline. The temporal dependence of the correlation curves are of interest for noise source modeling. Interestingly, data from the shear layer and the centerline show marked differences; the former shows significant oscillation which is absent in the latter. Many jet noise prediction schemes (refs. 8 and 10) model the temporal dependence by either an exponential decay: $\exp \left(-\tau / \tau_{0}\right)$ or a Gaussian decay: $\exp \left(-\tau^{2} / \tau_{0}^{2}\right)$ function. Clearly the centerline data shows better compliance to this modeling assumption than the shear layer counterpart. Additionally a closer scrutiny of the data shows that profiles become more asymmetric as the separation between the probe locations is increased.

To calculate the integral length scale in a fixed frame of reference (eq. 5) correlation coefficients at $\tau=0$ need to be plotted as a function of the probe separation $\eta_{\mathrm{x}}$. Figures $5(\mathrm{~b})$ and (d) show two such plots from two reference locations. Determination of the integral length scale involves calculating the area under the $r-\eta$ curve. While that is feasible for the centerline data, the negative oscillation in lip line data of figure 4(b) is expected to produce very small integral length scale. A length scale is a measure of the spatial extent of an eddy. In the lip shear layer, correlations persist over longer separations, indicating longer length scales; not the small value expected from figure 4(b). Therefore, calculations of the integral length scale in a fixed frame of reference were not pursued. This problem is avoided in calculations made in a moving frame of reference via the use of coherence spectra. The advantages of the frequency dependent scales were used in the recent noise modeling efforts of Goldstein and Khavran (ref. 24). 

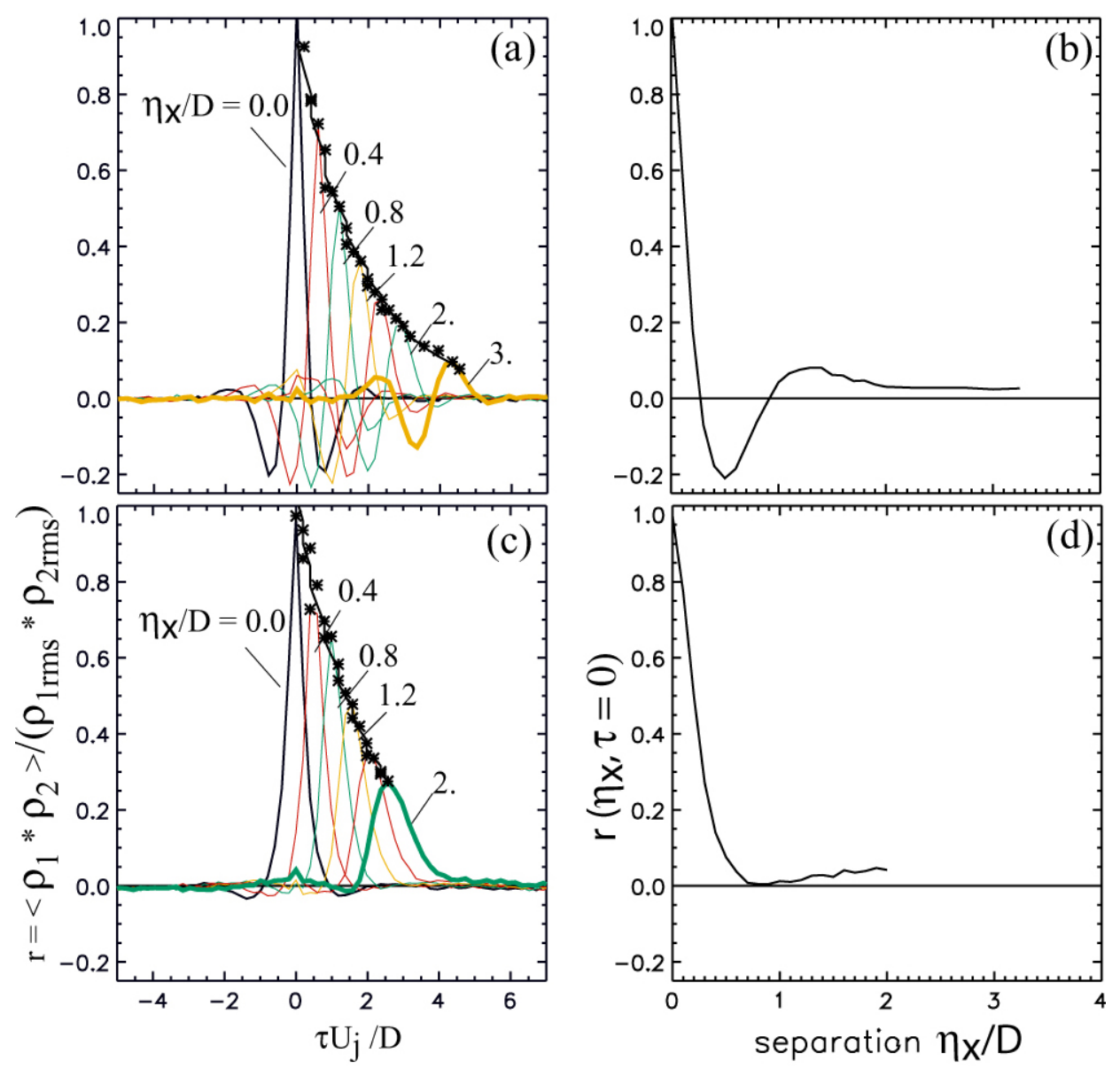

Figure 5.-(a), (c) Time domain correlation with the indicated probe separation and (b), (d) variation of the correlation coefficients at zero time separation from two different reference locations in $\mathrm{M}_{\mathrm{j}}=0.95$ jet. The reference probe is at (a), (b) lip line $\mathbf{X}(3 \mathrm{D}, 0.5 \mathrm{D})$ and (c), (d) centerline $\mathbf{X}(8 \mathrm{D}, 0 . \mathrm{D})$.

Envelopes of the correlation curves in figures 5(a) and (c) show the decay in the turbulent density fluctuation observed in a moving frame of reference. The integral time scale (in the moving frame) can be calculated from this envelope, representing the auto-correlation function in a moving frame, using equation (6). An exponential decay function was used to fit each envelope and the integral time scale $\mathrm{T}_{\tau}$ was calculated. The normalized time scales are presented in table I as "wideband" values. The time domain correlation data of figure 4 does not explicitly show frequency dependence of integral scales; $\mathrm{T}_{\tau}$ provides an average property over all frequencies. The time separation between the peak values of the correlation is a measure of the average convection time for an eddy to move from one probe location to the other. This allows for an estimation of the convective velocity, which once again is an average of all frequencies of fluctuation. Such wideband convection velocities are shown in table II. After knowing $U_{c}$ the wideband integral length scale in a moving frame can be calculated from the integral time scale: $L_{x_{\_} \text {wide }} / D=T_{\tau} U_{c} / D$. This is also shown in table I. 
TABLE I.-INTEGRAL SCALES IN MOVING FRAME OF REFERENCE

\begin{tabular}{|l|l|l|c|c|c|c|c|c|c|c|}
\hline Mj & \multicolumn{7}{|c|}{$\begin{array}{c}\text { Reference probe } \\
\text { location }\end{array}$} & $\begin{array}{c}\text { Time scale } \\
\mathrm{T}_{\tau} \mathrm{Uj} / \mathrm{D}\end{array}$ & \multicolumn{7}{|c|}{ Integral length scale $\mathrm{L}_{\mathrm{x}} / \mathrm{D}$} \\
\hline & $\mathrm{x} / \mathrm{D}$ & $\mathrm{r} / \mathrm{D}$ & Wideband & Wideband & $\mathrm{St}=0.3$ & 0.7 & 1.0 & 1.6 & 2.5 & 3.5 \\
\hline 0.95 & 3.0 & 0.5 & 1.29 & 0.9 & 2.88 & 1.44 & 0.67 & 0.41 & & \\
\hline 0.95 & 8.0 & 0 & 1.53 & 1.18 & 2.01 & 0.99 & 0.85 & 0.65 & & \\
\hline 1.4 & 3.0 & 0.5 & 1.22 & 0.88 & 3.01 & 1.81 & 1.16 & 0.46 & 0.33 & \\
\hline 1.4 & 10.0 & 0 & & & 1.84 & 1.03 & 0.84 & 0.69 & & \\
\hline 1.8 & 3.0 & 0.5 & & & 1.85 & 2.12 & 1.38 & 0.7 & 0.44 & 0.33 \\
\hline 1.8 & 12.0 & 0 & & & 2.2 & 1.07 & 0.85 & 0.65 & 0.48 & 0.43 \\
\hline 0.95 & 3.0 & 0.4 & & & 3.27 & 2.26 & 1.19 & 0.76 & & \\
\hline 0.95 & 3.0 & 0.6 & & & 2.15 & 0.93 & 0.45 & & & \\
\hline
\end{tabular}

TABLE II.-CONVECTIVE MACH NUMBER: $\mathrm{M}_{\mathrm{c}}=\mathrm{U}_{\mathrm{c}} / \mathrm{U}_{\mathrm{j}}$

\begin{tabular}{|c|c|c|c|c|c|c|c|c|c|}
\hline $\mathrm{Mj}$ & \multicolumn{7}{|c|}{$\begin{array}{c}\text { Reference probe } \\
\text { location }\end{array}$} & \multicolumn{7}{|c|}{ Convective Mach no. $\mathrm{M}_{\mathrm{c}}$} \\
\hline & $\mathrm{x} / \mathrm{D}$ & $\mathrm{r} / \mathrm{D}$ & Wideband & $\mathrm{St}=0.3$ & 0.7 & 1.0 & 1.6 & 2.5 & 3.5 \\
\hline 0.95 & 3.0 & 0.5 & 0.7 & 0.63 & 0.72 & 0.75 & 0.74 & & \\
\hline 0.95 & 8.0 & 0. & 0.77 & 0.73 & 0.78 & 0.82 & 0.88 & & \\
\hline 1.4 & 3.0 & 0.5 & 0.72 & 0.74 & 0.77 & 0.78 & 0.76 & 0.77 & \\
\hline 1.4 & 10.0 & 0 & & 0.76 & 0.81 & 0.83 & 0.88 & & \\
\hline 1.8 & 3.0 & 0.5 & & 0.66 & 0.77 & 0.8 & 0.82 & 0.86 & 0.86 \\
\hline 1.8 & 12.0 & 0 & & 0.74 & 0.8 & 0.83 & 0.88 & 0.9 & \\
\hline 0.95 & 3.0 & 0.4 & & 0.67 & 0.75 & 0.78 & 0.76 & & \\
\hline 0.95 & 3.0 & 0.6 & & 0.58 & 0.7 & 0.62 & & & \\
\hline
\end{tabular}

As described in the Introduction, the first step towards calculating frequency dependent scales is to determine auto- and cross- and coherence R (eq. 12) spectra. Figure 6 shows typical outcome. Physically, the $\mathrm{R}$ spectrum shows the frequency dependence of the correlation coefficient. The commonly used time-domain correlation data is unable to show this dependence. An examination of figure 6(b) shows that fluctuations around $\mathrm{St}=0.6$ remain well correlated $(\mathrm{R}=0.9)$ after $0.5 \mathrm{D}$ separation while those around $\mathrm{St}=2.5$ are less correlated $(\mathrm{R}=0.3)$. Following is a discussion of the measurement uncertainty. The calculation of the coherence spectrum involves a division process which poses a problem with experimental data. The inevitable random noise present in data sets is greatly amplified in the final result. To reduce the impact, the auto- and cross-spectra were first smoothened by applying a Savitzky-Golay filter (ref. 1) before calculating the coherence spectrum. The uncertainty in $\mathrm{R}$ is relatively small in the well converged part of the crossspectrum. As the separation between the probe volumes is increased the higher frequency part becomes progressively less correlated. This, at first, is reflected as an increase in the random error in the tail end of the
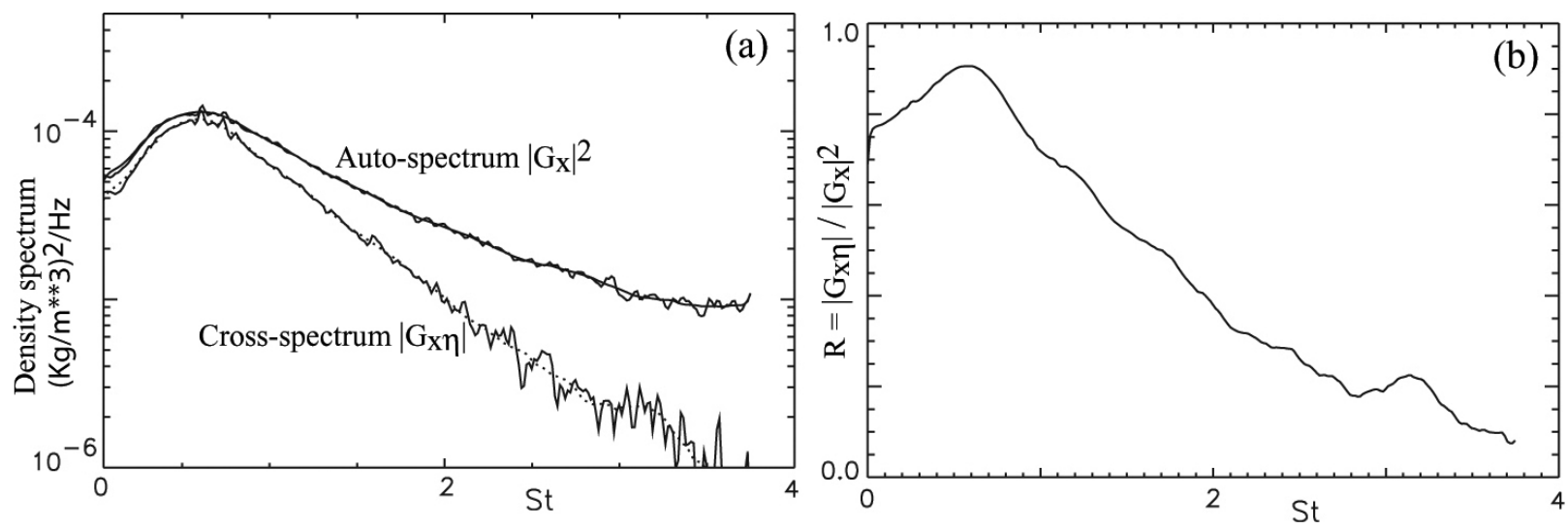

Figure 6.- (a) Auto spectrum of turbulent density fluctuations at $\mathbf{X}(3.0 \mathrm{D}, 0.5 \mathrm{D})$ and cross-spectrum from one probe at the same reference location and the other separated axially by $0.5 \mathrm{D}$ in $\mathrm{M}_{\mathrm{j}}=1.4$ jet. The dotted line show the smoothened spectra which was used to calculate coherence spectrum $\mathrm{R}$ shown in (b) using equation (10). 
cross-spectrum. With increasing probe separation the high frequency part becomes submerged in the experimental noise floor. For these instances the coherence spectrum flattens out, and frequently starts to lift up at the high-frequency end. To avoid larger error the frequency range for which $\mathrm{R}<0.2$ was not used for the subsequent analysis.

Coherence spectra for increasing probe separation are shown in figure 7. For the most part such spectra decay with increasing probe separation. The rate of reduction is faster with increasing frequency, which reflects a shorter length scale for higher frequency eddies. The frequency range $0.15 \leq \mathrm{St} \leq 0.8$ in the shear layer data (fig. 7(a)), however; shows lasting correlation even when the probes are separated by four diameters. Frequencies above and below this range show quicker decay. The Kelvin-Helmholtz instability waves present at the jet shear layer are expected to be in the above frequency range. The increased correlation is reflective of longer length scales associated with these instability waves. At the centerline and beyond the end of the potential core the coherent structures are of lower frequency. This is reflected in R spectra shown in figure 7(b).

Coherence spectra are useful in calculating frequency dependent length scales. Towards this end $\mathrm{R}$ values for desired frequencies and for all probe separations were extracted and plotted in figures 8(a) and (c). Unlike the time-domain correlation $\mathrm{r}$, the frequency domain coherence $\mathrm{R}$ does not become negative, therefore; problems associated with calculating integral length scale are avoided. Note that for certain frequencies coherence values become greater than unity. This is indicative of a growing level of fluctuation due to the turbulence production as discussed after equation 10. Nevertheless, $\mathrm{R}>1$ does not affect integral length scale calculations. An exponential curve $\mathrm{R}=\mathrm{c}_{1} \exp \left(-\mathrm{c}_{2} \eta_{\mathrm{x}}\right)$ was fitted through data sets for each frequency; $\mathrm{c}_{1}$ and $\mathrm{c}_{2}$ are fitted constants. To reduce the level of uncertainty data points close to the noise floor were left out of the curve fitting process. The integral length scale was determined as:

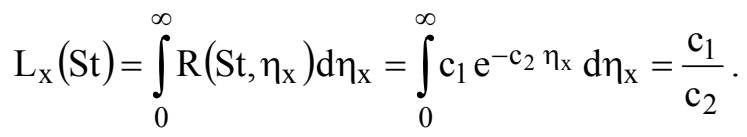

The calculated values for the integral scales for different reference probe location are presented in table I. An examination of data presented in this table provides many interesting observations. (a) As expected the integral length scale decreases with increasing Strouhal frequency. In other words, smaller eddies have higher frequencies. (b) The wideband length scale, traditionally used in noise source modeling, is representative of the mid-frequency range $0.7<\mathrm{St}<1.0$. (c) At the high frequency end, $\mathrm{St}>2$, length scales are similar in the shear layer and the centerline, this perhaps points towards a universal behavior of the high frequency eddies. However, at the low frequency end $\mathrm{St}<1.0$ lip shear layer scales are longer than the centerline ones. (d) A rapid decrease in the length scale is observed radially across the lip shear layer.
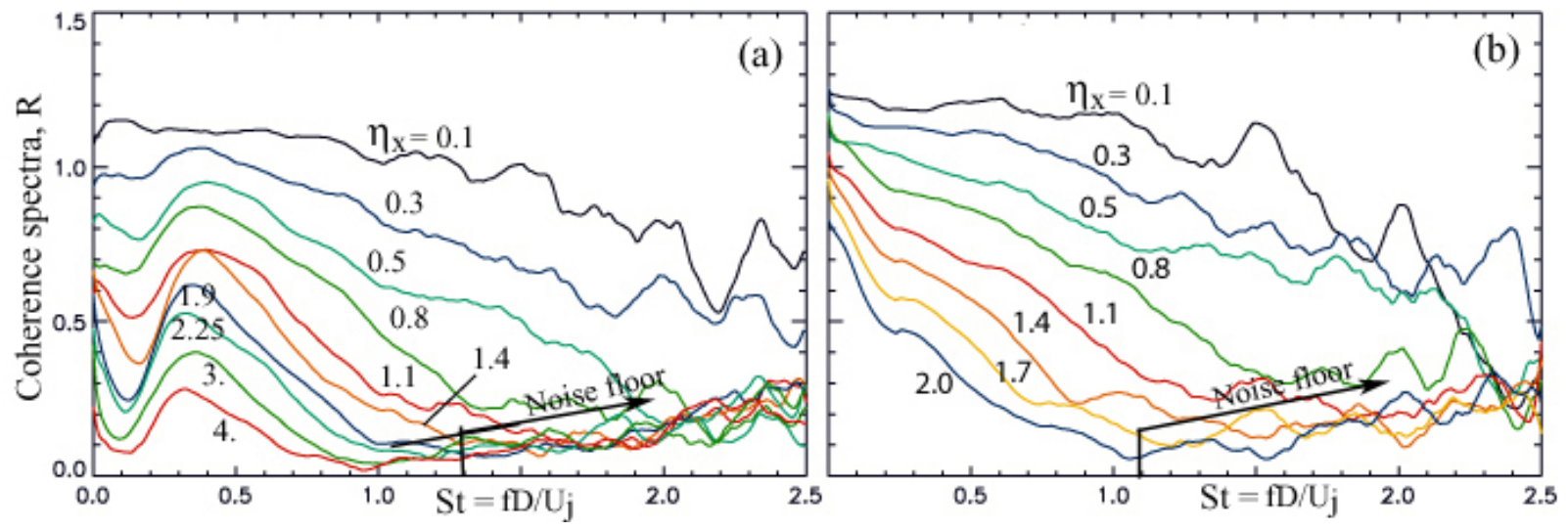

Figure 7.- Variation of coherence spectra with increasing (indicated) probe separation. The reference probe is at (a) lip line $\mathbf{X}(3 \mathrm{D}, 0.5 \mathrm{D})$, and (b) centerline $\mathbf{X}(8 \mathrm{D}, 0 . \mathrm{D})$ of $\mathrm{Mj}=0.95$ jet. 


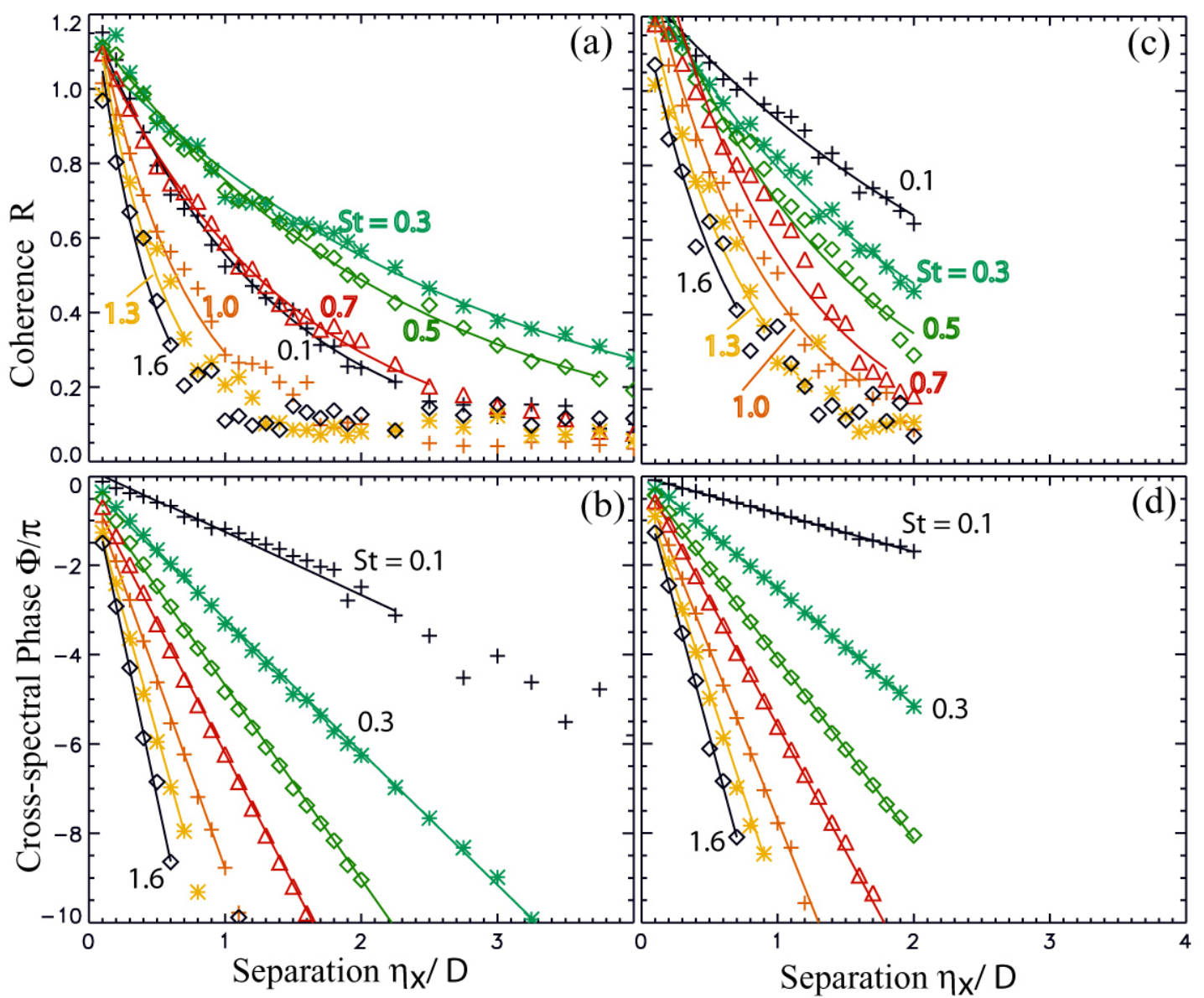

Figure 8.-Variation of coherence and cross-spectral phase with probe separation. Data from the same locations as in the earlier figure; $\mathrm{Mj}=0.95$, fixed probe at (a), (c) lip line $\mathbf{X}(3 \mathrm{D}, 0.5 \mathrm{D})$, and (b), (d) centerline $\mathbf{X}(8 \mathrm{D}, 0 . \mathrm{D})$.

Returning back to figures $8(\mathrm{~b})$ and (d), at a given frequency, the variation of the cross-spectral phase with spatial separation provides convective velocity (eq. 8) at that frequency. A linear fit through data points from different probe separations was used to reduce random error. The frequency dependent convective Mach numbers $\mathrm{M}_{\mathrm{c}}(\mathrm{St})=\mathrm{U}_{\mathrm{c}} / \mathrm{U}_{\mathrm{j}}$ are shown in table II. An examination of this table shows the following. (a) The higher the Strouhal frequency the higher the convective Mach number. This is intuitively expected as the smallest eddies are expected to be convected close to the mean flow velocity. (b) $\mathrm{M}_{\mathrm{c}}$ is higher for fluctuations present in the centerline than at the lip line. This perhaps is a reflection of the higher mean velocity at the jet centerline. (c) $\mathrm{M}_{\mathrm{c}}$ decreases radially away from the jet centerline.

The frequency and Mach number dependencies are further explored in figure 9. The length scales are normalized by Strouhal frequency $\mathrm{L}_{\mathrm{x}} \mathrm{St} / \mathrm{D}$, yet the frequency dependence can not be totally eliminated. In general, this normalized length scales increases with frequency before attaining a near constant level. The same is true with the convective velocity. The shear layer data in figure 9(a) shows a low frequency range $(\mathrm{St}=0.3$ to 1.0$)$ where length scales are unusually longer. The range varies with Mach number, nevertheless the longer length scales are indicative of Kelvin-Helmholtz instability waves which stay coherent over longer distances. The centerline data in figures 9(c) and (d) shows collapse of data points from all three jets under study. Note that reference points were selected such that they are approximately 

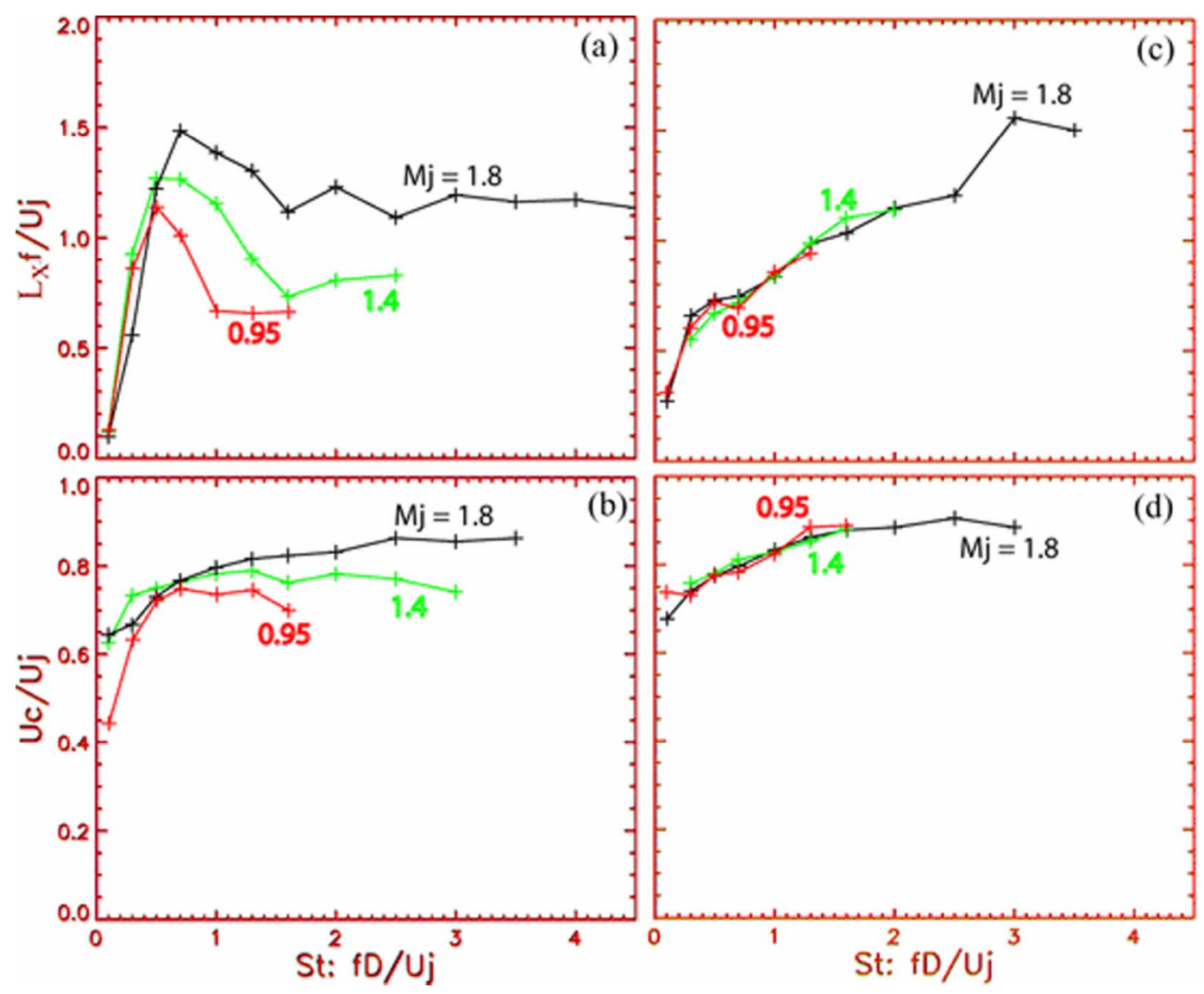

Figure 9.- - Variation of (a), (c) moving frame integral length scales and (b), (d) convective Mach number with Strouhal frequency in three different Mach number plumes. In (a) and (b) the reference probe was kept fixed at a shear layer position of $\mathbf{X}(3 \mathrm{D}, 0.5 \mathrm{D})$; while in (c) and (d) it was moved to (8D, 0.D), (10D, 0.D), and (12D, 0.D) in $\mathrm{Mj}=0.95,1.4$, and 1.8 jets respectively.

1.2 times the potential core length away from the nozzle exit. The potential core length is a measure of the compressibility effect. The collapse of data shows that it should be used to scale the Mach number dependence of integral length scales. Note that the experimental uncertainty in $\mathrm{L}_{\mathrm{x}}$ at the highest resolved frequency is relatively higher than the lower frequency points. This is caused by a fast decay in coherence within a relatively small probe separation. Hence, only a few separation points can be used to estimate the high frequency length scales.

Finally, figure 10 presents wave-number frequency spectrum from reference points at lip shear layers and centerlines of two different Mach number jets. For each reference point $\mathbf{X}$, cross-correlation data were measured from $n=64$ equally spaced, $\Delta \eta_{x}=0.1 \mathrm{D}$, probe separations (including the overlapped probe location) along the axial direction. The complex cross-spectrum $G_{x \eta}\left(\mathbf{X}, \eta_{x}, \omega_{1}\right)$ was calculated for each probe separation following equation (20) $\left(\omega_{1}=2 \pi f_{1}\right)$. Cross-spectral data for each frequency $\omega_{1}$ were extracted for all probe separations and a spatial Fourier transform was performed:

$$
\mathrm{S}_{\mathrm{k} \omega}\left(\times, \mathrm{k}_{\mathrm{x}, \mathrm{m}}, \omega_{1}\right)=\sum_{\mathrm{j}=0}^{\mathrm{n}-1} \mathrm{G}_{\mathrm{x} \eta} \exp \left(\mathrm{j} \frac{2 \pi \mathrm{im}}{\mathrm{n}}\right), \quad \mathrm{k}_{\mathrm{x}, \mathrm{m}}=\frac{\mathrm{m}}{\mathrm{n} \Delta \eta_{\mathrm{x}}} \quad \mathrm{m}=-\frac{\mathrm{n}}{2}, \ldots .+\frac{\mathrm{n}}{2}
$$




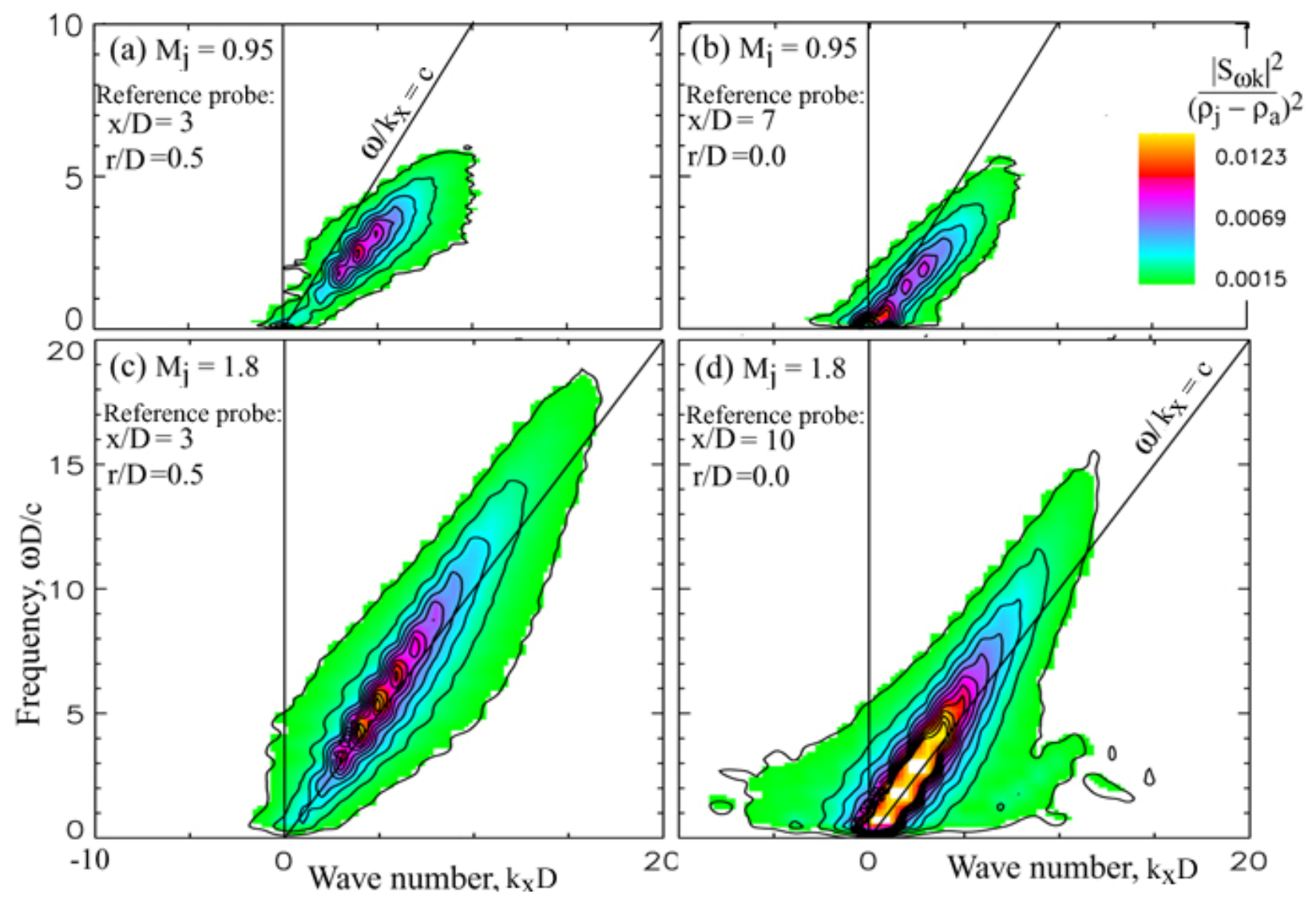

Figure 10.- Magnitude of the frequency-wave number spectrum measured from the indicated probe locations in the indicated Mach number plumes. The diagonal lines correspond to a convective velocity equal to the ambient sound speed.

The subscript $\mathrm{x}$ in $\mathrm{k}_{\mathrm{x}, \mathrm{m}}$ denotes the axial wave number which is evaluated at $\mathrm{m}$ different points. The magnitude of each spectrum, normalized by $\left(\rho_{\mathrm{j}}-\rho_{\mathrm{a}}\right)^{2}, \rho_{\mathrm{a}}$ is ambient density and $\rho_{\mathrm{j}}$ is the core density, is shown in figure 10. The staircase like appearance of the color plots is reflective of the coarse wave number resolution, $\Delta \mathrm{k}_{\mathrm{x}}=1.56$, achievable with the limited spatial data. Additionally, the minimum value in the color scale was selected to avoid plotting the noise floor. The utility of the k- $\omega$ spectrum to directly determine the radiative component of turbulent fluctuations was discussed earlier in the introduction. The part of the spectrum that lies to the left of the sonic speed line $\omega / \mathrm{k}_{\mathrm{x}}=\mathrm{c}_{\infty}$ is capable of radiating at different observer angles. While most of the fluctuations in the subsonic Mach 0.95 jet are incapable of radiating to the far field, the supersonic Mach 1.8 jet shows a different scenario. With an average supersonic convective velocity, fluctuations in both the lip shear layer and the centerline of the jet are expected to radiate strongly along $\theta=\cos ^{-1}\left(\mathrm{c}_{\propto} / \mathrm{U}_{\mathrm{c}}\right) \sim 20^{\circ}$ to $25^{\circ}$ to the jet axis. The figure identifies that some parts of the low frequency fluctuations in both jets are capable of radiating at all angles. In this regard, compared to the shear layer, fluctuations from the centerline of the jet are found to possess larger radiative component of fluctuations.

\section{Summary and Conclusion}

Two point space-time correlations of density fluctuations were measured in subsonic and supersonic unheated plumes using a non-invasive Rayleigh scattering based optical diagnostics technique. The density fluctuations in the unheated plumes are solely due to the compressibility effect, and are expected 
to be closely related to the noise sources. The two-laser beam Rayleigh scattering setup allowed pointmeasurements from two independently located probe positions in the plume. The system was designed and built from scratch using available laser, optical components, and electronics. Photo-electron counting electronics were used to measure the intensities of the scattered light collected from probe volumes. The count rates were directly related to the local air densities. To measure time history of fluctuating air density, counting was performed in contiguous time bins over long durations. Analysis of the count signals provided various statistics of space-time correlations. The particular goal of the present work was to determine the frequency dependent correlation scales in high velocity jets which were unavailable till now. Following is a summary of the primary observations:

(a) Correlations were measured from the lip shear layer as well as from centerline and downstream of the potential core. The two sets show significant differences. At longer time delays significant negative correlations were measured in the shear layer data. This was mostly absent from the centerline data. The negative correlation prohibited realistic calculations of integral length scales in a fixed frame of reference. The time envelope of correlations from many different probe separations provided moving frame integral time scale and convective velocity. These values provide "wideband" or average property of all frequencies of turbulent fluctuations.

(b) To obtain frequency dependent correlation, cross-correlation data were Fourier transformed and normalized by fluctuations measured at the overlapped probe location. The coherence spectrum provided frequency dependent correlation coefficients, while the phase of the cross-spectrum provided measures of convective velocities. As expected the convection velocity is found to increase with fluctuations frequency. The variation of the magnitude of coherence spectra with probe separation allowed for calculations of the frequency dependent length scales $\mathrm{L}(\mathrm{St})$ in a moving frame of reference. Such length scales were found to decrease progressively with frequency.

A closer look into the shear layer data shows a range of frequencies, typically $0.1 \leq \mathrm{St} \leq 1.0$ (the range varied with Mach number and probe location) where integral length scales are longer than the neighboring frequencies. The longer length scales are believed to be associated with the Kelvin-Helmholtz instability waves.

(c) Data obtained from different Mach number jets were found to collapse when the reference probe locations were varied according to the length of the potential core.

(d) By taking correlation data from a large number of spatial separations and by taking spatial Fourier transforms of cross-spectral data sets, the frequency-wave number $\omega-\mathrm{k}$ spectrum was constructed. A comparison of such spectra from different Mach number jets showed a significant increase in the radiative part of turbulent fluctuations in supersonic jets. For all Mach number jets low frequency fluctuations from the centerline of the jet, compared to that in the shear layer, are found to possess larger radiative components.

\section{References}

1. Townsend, A.A. "The structure of turbulent shear flows," Cambridge University Press, Cambridge, UK, 1976.

2. Goldstein, M.E. 1976 Aeroacoustics, McGraw-Hill International Book Co., New York 564-587.

3. Tam, C.K.W., and Auriault, L., "Jet Mixing Noise from Fine-Scale Turbulence," AIAA Journal, vol. 37, no. 2, 1999, pp. 145-153.

4. Laurence, J.C. 1956 "Intensity, scale and spectra of turbulence in mixing region of free subsonic jet," NACA rep. no. 1292

5. Davies, P.O.A.L., Fisher, M.J., and Barratt, M.J. "The characteristics of turbulence in the mixing region of a round jet," J. Fluid Mech., 15, 1962, pp. 337-367.

6. Tam, C.K.W., Golebiowski, M. and Seiner, J.M. "On the Two Components of Turbulent Mixing Noise from Supersonic Jets," AIAA paper 96-1716, 1996.

7. Harper-Bourne, M. "Jet noise turbulence measurements," AIAA paper 2003-3214.

8. Khavaran, A. and Bridges, J. "Modeling of turbulence generated noise in jets," AIAA-2004-2983. 
9. Bridges, J. and Wernet, M. "Measurements of the aeroacoustic sound source in hot jets," AIAA-2003-3130.

10. Hunter, C.A. and Thomas, R.H. "Development of a Jet Noise Prediction Method for Installed Jet Configurations," AIAA-2003-3169.

11. Fisher, M.J. and Davies, P.O.A.L., "Correlation measurements in a non-frozen pattern of turbulence," J. Fluid Mech., 18, 1964, pp. 97-116.

12. Jordan, P. and Gervais, Y. "Modeling self and shear noise mechanisms in anisotropic turbulence," AIAA paper no. 2003-3318.

13. Kerherve, F., Jordan, P., Gervais, Y. and Valiere, J.-C.,"Aerodynamic characterization of a supersonic jet using two-point laser Doppler velocimetry," AIAA paper 2003-3215, 2003.

14. Doty, M.J. and McLaughlin, D.K. "Space-time correlation measurements of high speed axisymmetric jets using optical deflectometry," Experiments in Fluids, 38, 415-425, 2005.

15. Debonis, J.R. and Scott, J.N. "Large-eddy simulation of a turbulent compressible round jet," AIAA J., vol. 40, no. 7, 2002, pp. 1346-1354.

16. Freund, J.B. "Noise sources in a low-Reynolds-number turbulent jet at Mach 0.9," J. Fluid Mech. (2001), vol. 438, pp. 277-305.

17. Panda, J., Seasholtz, R.G., Elam, K.A. and Mielke, A.F., "Time-averaged Velocity, Temperature and Density Surveys of Supersonic Free Jets," ASME paper HT-FED2004-56856, 2004 ASME Heat Transfer/Fluids Engineering Summer Conference, July 2004.

18. Panda, J. and Seasholtz, R.G. "Experimental investigation of density fluctuations in high-speed jets and correlation with generated noise," Journal of Fluid Mechanics, vol. 450, pp. 97-130, 2002.

19. Panda, J., Seasholtz, R.G. and Elam, K.A. "Further Progress in Noise Source Identification in High Speed Jets Via Causality Principle," Journal of Fluid Mechanics, August 2005.

20. Wills, J.A.B. "On convection velocities in turbulent shear flows," J. Fluid Mech., 20(3), 417-432, 1964.

21. Willmarth W.W. and Woolridge C.E. J. Fluid Mech., vol. 14, 1962 pp. 187-

22. Goldstein, M.E. "On identifying the true sources of aerodynamic sound," J. Fluid Mech., 526, 337-347, 2005.

23. Crighton, D.G. "Basic principles of aerodynamic noise generation" Prog. Aerospace Sci. 16, 1975, pp. 31-96.

24. Goldstein, M.E. and Khavran A., "Acoustic source modeling for high speed air jets," AIAA paper 2005-415.

25. Press W.H., Flannery, B.P., Teukolsky, S.A., and Vetterling, W.T. Numerical Recipes in Fortran Cambridge University Press, 1992. 

